Physics

Physics Research Publications

\title{
Femtosecond response of diffraction efficiency of GaAs/AlGaAs photorefractive multiple quantum well
}

H. Tanaka*

S. Tsuboi**

O. Wada ${ }^{\S}$

K. Terawaki ${ }^{\dagger}$

S. Nagahara ${ }^{\dagger \dagger}$

K. Nakagawa ${ }^{\text {I }}$

K. $\mathrm{Kou}^{\ddagger}$

T. Kita ${ }^{\ddagger \ddagger}$

D. D. Nolte" 


\title{
Femtosecond Response of Diffraction Efficiency of GaAs/AlGaAs Photorefractive Multiple Quantum Well
}

\author{
Hirokazu Tanaka, Kouji Terawaki, Kazukuni Kou, Satoshi Tsuboi, Seiji Nagahara,
} Takashi Kita, Osamu Wada, Kiyoshi NaKagawa ${ }^{1}$ and David D. Nolte ${ }^{2}$

Department of Electrical and Electronic Engineering, Kobe University, 1-1 Rokkodai, Nada, Kobe 657-8501, Japan

${ }^{1}$ Department of Reliability-Based Information Systems Engineering, Kagawa University, 2217-20 Hayashi-cho, Takamatsu 761-0369, Japan

${ }^{2}$ Department of Physics, Purdue University, West Lafayette, Indiana 47907-1396, USA

(Received September 18, 2002; accepted for publication November 5, 2002)

Ultrafast response of the diffraction efficiency of a photorefractive multiple quantum well (PRMQW) has been investigated by pump-probe spectroscopy. We evaluated the temporal evolution of the diffraction efficiency of the PRMQW using a differential transmission spectrum. The results were compared with the output-widening factor of diffracted pulses. A good agreement obtained between our evaluation results and the results of electric-field cross correlation has confirmed the applicability of our technique in characterizing the diffraction efficiency of the PRMQW. [DOI: 10.1143/JJAP.42.2329]

KEYWORDS: photorefractive material, quantum well, diffraction efficiency, pump-probe technique, pulse shaper, optical gate, femtosecond pulse

\section{Introduction}

Photorefractive quantum well (PRQW) devices are attractive for applications such as pulse shapers and optical gates because of their high sensitivity, small saturation intensity and short response time. ${ }^{1-6)}$ Pulse shapers use spatial control of a spectrally dispersed light signal and all optical gates use ultrafast diffraction because of optically induced gratings. In these applications, not only an ultrafast response time but also a broad diffraction bandwidth is of great importance. In the case of using the PRMQW devices in a dynamic spectral holography system with a $100 \mathrm{fs}$ ultrashort pulse, the bandwidth must cover approximately $10 \mathrm{~nm}$. To characterize such PRMQW with a broad diffraction bandwidth, time-resolved spectroscopy of the diffraction efficiency spectrum is prerequisite. In this paper, we report a technique of evaluating the dynamics of the diffraction efficiency in the PRMQW using femtosecond pulses and discuss the applicability of our technique in characterizing the diffraction efficiency of the PRMQW.

\section{PRMQW Device Structure and Experiments}

The PRMQW device structure used in this study is shown in Fig. 1. The growth was performed by molecular beam epitaxy. A $500 \mathrm{~nm}$ buffer layer of GaAs was grown on a semi-insulating GaAs (001) substrate. After the buffer layer growth, $500 \mathrm{~nm} \mathrm{Al}_{0.5} \mathrm{Ga}_{0.5} \mathrm{As}$ and $20 \mathrm{~nm}$ AlAs layer were grown as selective etching layers to remove the GaAs substrate. Then $200 \mathrm{~nm} \mathrm{Al}_{0.2} \mathrm{Ga}_{0.8} \mathrm{As}$ and $\mathrm{GaAs} / \mathrm{Al}_{0.3} \mathrm{Ga}_{0.7} \mathrm{As}$ MQW were grown on AlAs layer, which was followed by $100 \mathrm{~nm} \mathrm{Al}_{0.2} \mathrm{Ga}_{0.8}$ As and $5 \mathrm{~nm}$ GaAs. The top GaAs layer protects the device from oxidaton. The GaAs well width was $7 \mathrm{~nm}, \mathrm{Al}_{0.3} \mathrm{Ga}_{0.7} \mathrm{As}$ barrier width was $6 \mathrm{~nm}$ and the repetition was 100 periods. After the growth, the sample was protonimplanted at a dose of $10^{12} \mathrm{~cm}^{-2}$ at $160 \mathrm{keV}$ and at a dose of $5 \times 10^{11} \mathrm{~cm}^{-2}$ at $80 \mathrm{keV}$ to create deep-level defects and render the material semi-insulating and persistently photorefractive. The implanted wafer was cleaved and was bonded onto the glass plate with the epilayer side on the glass. To remove the substrate for transmission experiments, the substrate was polished by lapping to $100 \mu \mathrm{m}$ and etched to $\mathrm{Al}_{0.2} \mathrm{Ga}_{0.8} \mathrm{As}$. An $\mathrm{HF}$ acid etch was used to remove the $\mathrm{Al}_{0.2} \mathrm{Ga}_{0.8} \mathrm{As}$ and AlAs layer. Gold contacts were evaporated directly onto the GaAs layer with a $1 \mathrm{~mm}$ aperture. When we measured the electric-field cross correlation between an input pulse and a diffracted pulse, an electric field was applied in the plane of the MQW.

We measured the transmission spectra of this device with increasing central wavelength of incident pulses. Pulses used in this experiment were generated by a Ti:sapphire laser for $100 \mathrm{fs}$ and a repetition rate of $80 \mathrm{MHz}$. Pump and probe excitation densities were 240 and $40 \mathrm{fJ} / \mu^{2}$, respectively.

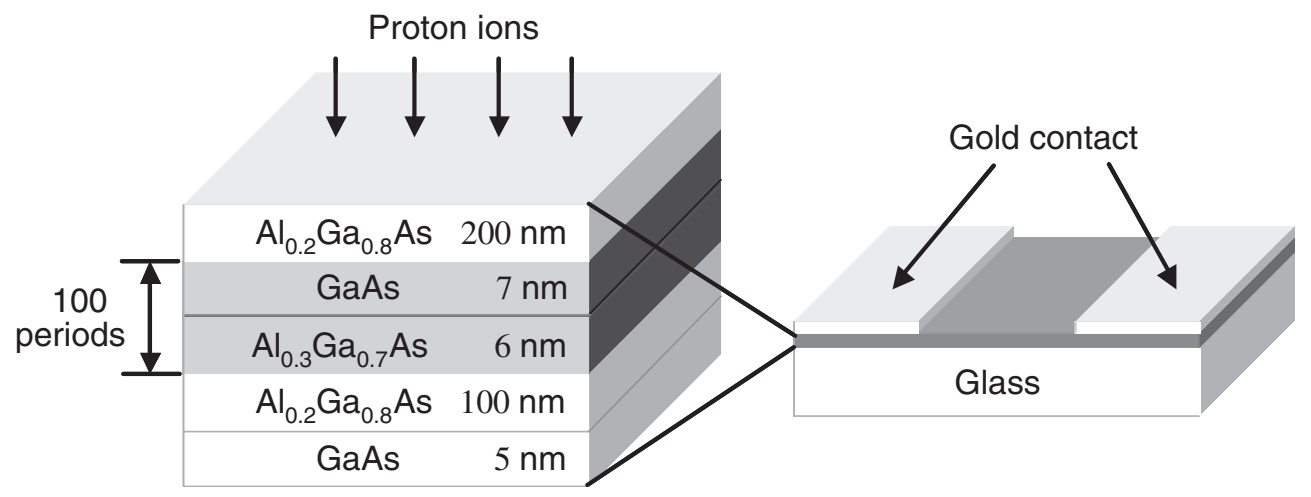

Fig. 1. PRMQW device structure. 


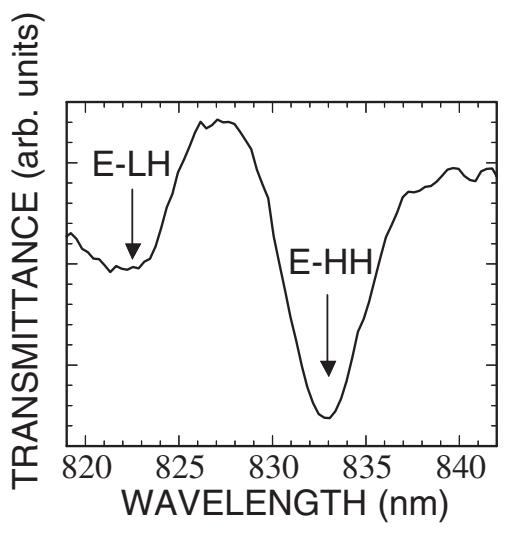

Fig. 2. Transmission spectrum.

The incident pulses with an approximately Gaussian shape had a bandwidth of $8 \mathrm{~nm}$. The integrated transmission spectrum is shown in Fig. 2. Two resonant absorption lines are observed at $\sim 833 \mathrm{~nm}$ and $\sim 823 \mathrm{~nm}$, which correspond to absorption resonances of the E-HH (electron-heavy hole) and E-LH (electron-light hole) exciton transitions, respectively.

\section{Ultrafast Evolution of Diffraction Efficiency}

We measured differential transmission $\Delta T / T$ of the PRMQW by using the pump-probe technique with increasing central wavelength from $820 \mathrm{~nm}$ to $842 \mathrm{~nm}$, which covers the E-HH and E-LH exciton transitions. We show the results in Fig. 3. Ultrafast responses of $\sim 200 \mathrm{fs}$ are observed at each wavelength. In particular, the positive signal in the $\Delta T / T$ response is enhanced near the E-HH and E-LH exciton transitions because of exciton bleaching and the spectral hole. ${ }^{7)}$

The measured $\Delta T / T$ can be converted into an output diffraction efficiency spectrum $\eta_{\text {out }}(\lambda)$ at a given delay time. The output diffraction efficiency is

$$
\eta_{\text {out }}(\lambda)=\left(\frac{d}{4} \Delta \alpha(\lambda)\right)^{2}+\left(\frac{\pi d}{\lambda} \Delta n(\lambda)\right)^{2},
$$

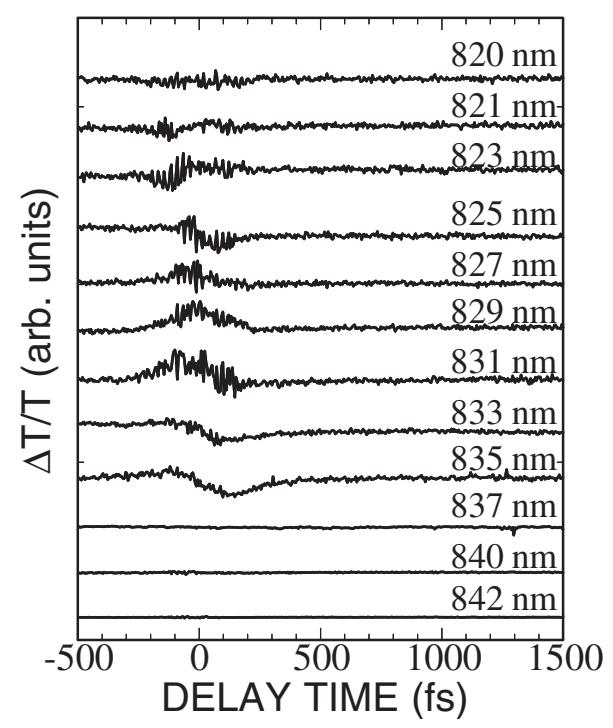

Fig. 3. $\Delta T / T$ measured at various wavelengths.

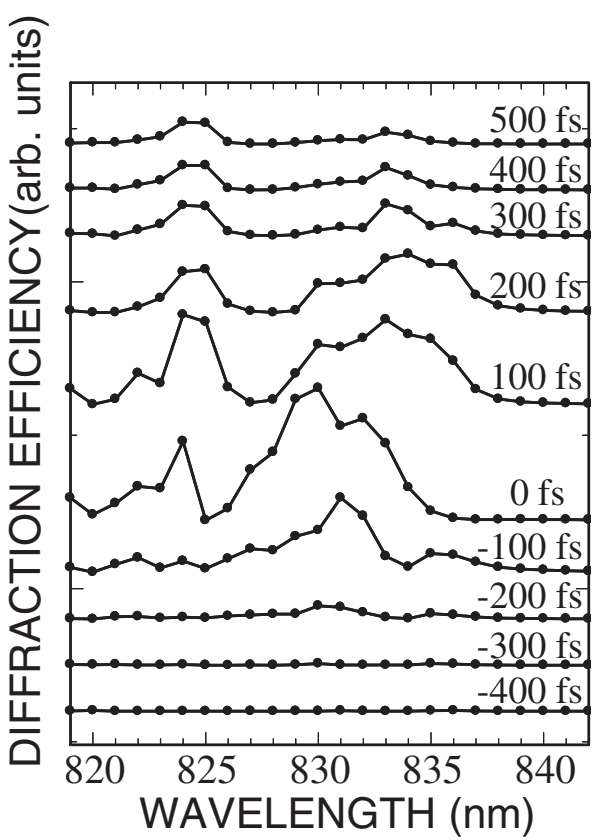

Fig. 4. Temporal evolution of diffraction efficiency evaluated from $\Delta T / T$.

where $d$ is the thickness of the device. ${ }^{2)}$ For a small pumpinduced absorption change $(-\Delta \alpha), \Delta T / T$ is regarded as $-\Delta \alpha d$. $^{7)}$ Using the Kramers-Kronig relation, $\Delta n$ is derived from $\Delta \alpha$. The obtained $\Delta \alpha(\lambda)$ and $\Delta n(\lambda)$ at a given delay time yield a $\eta_{\text {out }}(\lambda)$ spectrum. The diffraction efficiency spectra evaluated by this method as a function of the delay time are shown in Fig. 4. We succeeded in clearly resolving femtosecond dynamic responses of the diffraction efficiency due to the E-HH and E-LH exciton transitions. The peaks of the diffraction efficiency for the E-HH and E-LH exciton transitions shift slightly toward the lower energy side with increasing delay time. We consider that high-density photogenerated carriers redistribute in the QWs and induce band bending. The observed red-shift corresponds to the Stark effect in the QWs.

To characterize femtosecond pulse diffraction from the PRMQW, we performed degenerate four-wave mixing measurements. ${ }^{2)}$ The applied electric field was $10 \mathrm{kV} / \mathrm{cm}$. Typical results are shown in Fig. 5. The transmission spectrum for an input control wavelength of $833 \mathrm{~nm}$ shows a dip structure because of the E-HH exciton absorption. On the other hand, the diffracted bandwidth is $6 \mathrm{~nm}$. The slight narrowing of the bandwidth is caused by the nonflat diffraction efficiency. In order to characterize the time dependence of the diffracted and transmitted pulses, we used the electric-field cross-correlation between the signal pulse and a known reference pulse. Electric-field cross-correlation can be used to characterize the low diffracted pulse powers obtained in our experiments. Electric-field cross-correlations for the diffracted pulse and the transmitted pulse are shown in Fig. 5. The electric-field cross-correlation trace of the diffracted pulse is wider than that of the input pulse. This widening reflects the diffraction efficiency at $833 \mathrm{~nm}$. The full-widths at half maximum of the electric-field crosscorrelation of the diffracted pulse and the transmitted pulse are denoted by $\tau_{\mathrm{D}}$ and $\tau_{\mathrm{T}}$, respectively. We obtained a diffracted pulse duration $\Delta t_{\mathrm{D}}$ from $\tau_{\mathrm{D}}$ and a transmitted 


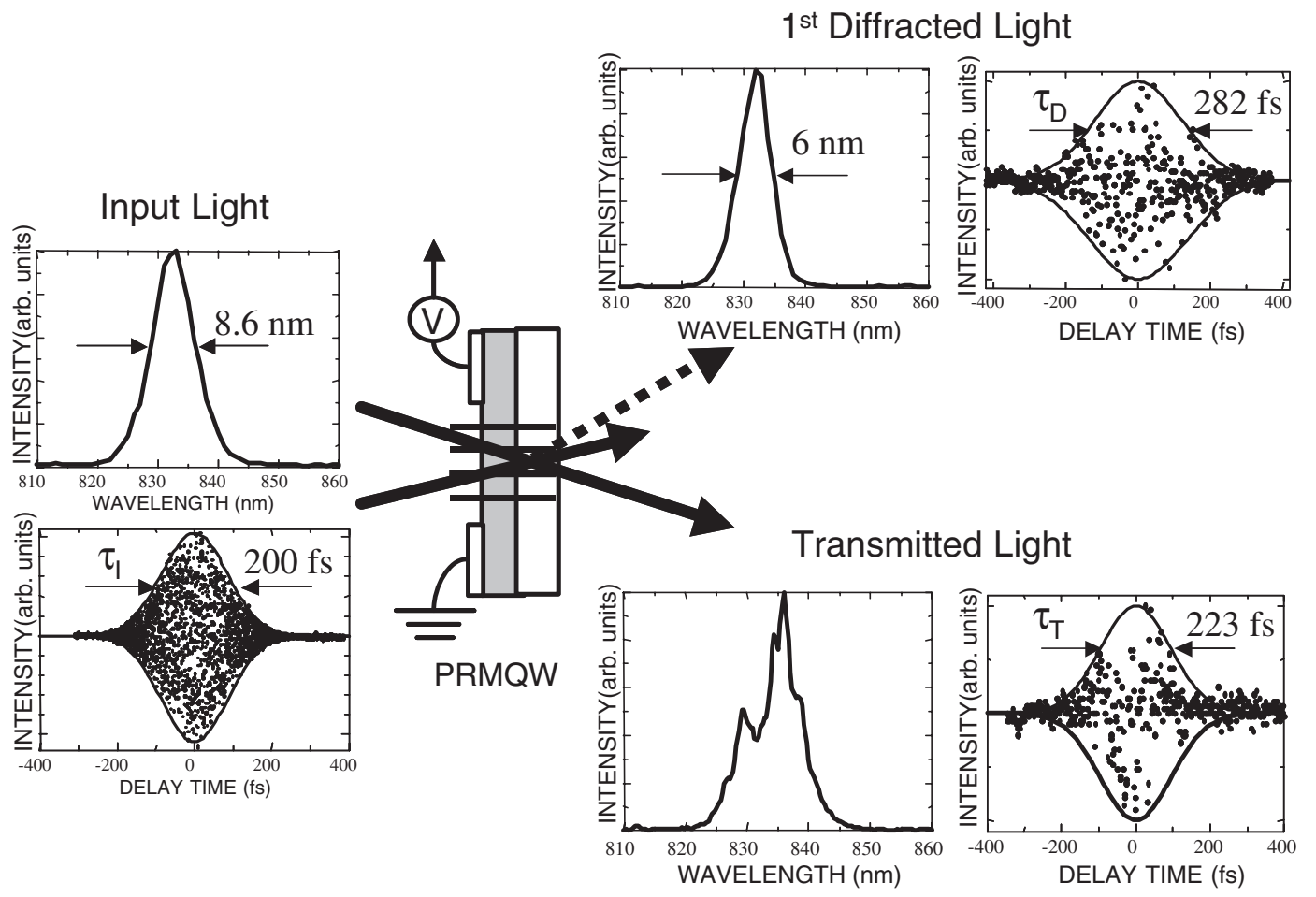

Fig. 5. Spectra and electric-field cross-correlation traces for incident, transmitted and diffracted pulses.

pulse duration $\Delta t_{\mathrm{T}}$ from $\tau_{\mathrm{T}}$ according to the following relation.

$$
\tau_{\mathrm{D} \text { or } \mathrm{T}}=\left[2\left(\Delta t_{\mathrm{D} \text { or } \mathrm{T}}^{2}+\Delta t_{\mathrm{ref}}^{2}\right)\right]^{1 / 2}
$$

$\Delta t_{\mathrm{T}} / \Delta t_{\mathrm{D}}$ is defined as the output-widening factor, which can be used as an index of the diffraction efficiency.

$\Delta t_{\mathrm{T}} / \Delta t_{\mathrm{D}}$ values for different input central wavelengths of $831,833,835$ and $837 \mathrm{~nm}$ are summarized in Fig. 6. The widening factors are compared with the diffraction efficiency spectrum evaluated from the pump-probe measurements (Fig. 5). The solid line in Fig. 6 shows the time-integrated diffraction efficiency spectrum. Here the spectra measured in a range from $-400 \mathrm{fs}$ to $500 \mathrm{fs}$ in Fig. 4 are integrated. A dashed line in Fig. 6 indicates the output-widening factor obtained from the electric-field cross-correlation. The

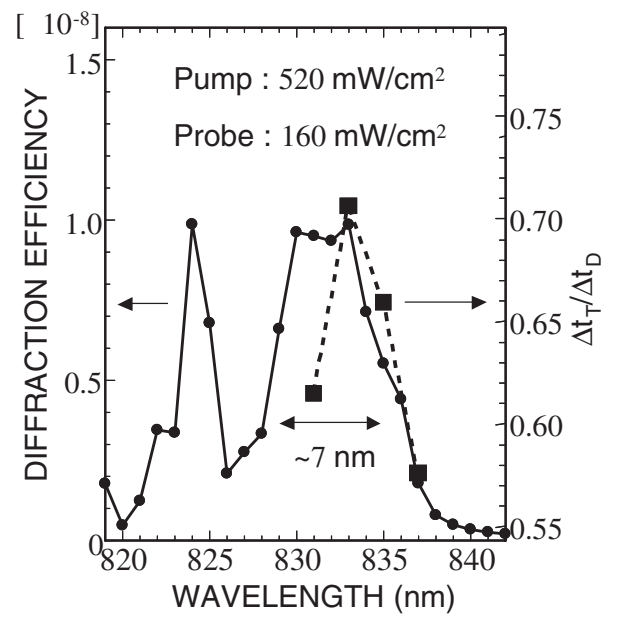

Fig. 6. Comparison between the evaluated time-integrated diffraction efficiency spectrum and the output-widening factors. diffraction bandwidth is about $7 \mathrm{~nm}$, which is sufficiently wide to pass a pulse shorter than $200 \mathrm{fs}$. We observe an agreement between the evaluated diffraction efficiency and the measured widening factor. This confirms the applicability of our technique in chracterizing the diffraction efficiency precisely.

\section{Conclusions}

We have systematically studied the diffraction efficiency of the PRMQW device in the femtosecond scale. We evaluated the diffraction efficiency from $\Delta T / T$ using the pump-probe technique. The diffraction bandwidth of our PRMQW device was measured to be $7 \mathrm{~nm}$, showing the usefulness of PRMQW for pulses less than $200 \mathrm{fs}$. The evaluated diffraction efficiency spectrum was compared with the output-widening factor obtained from the electric-field cross-correlation. The good agreement obtained between our evaluated results and the results of the electric-field crosscorrelation has confirmed the applicability of our technique in characterizing the diffraction efficiency of PRMQW.

1) R. M. Brubaker, Y. Ding, D. D. Nolte, M. R. Melloch and A. M. Weiner: IEEE J. Quantum Electron. 33 (1997) 2150.

2) M. Dinu, K. Nakagawa, M. R. Melloch, A. M. Weiner and D. D. Nolte: J. Opt. Soc. Am. B 17 (2000) 1313.

3) K. Nakagawa, T. Minemoto, T. Kita, K. Yamashita, T. Nishino, M. Dinu, D. D. Nolte and M. R. Melloch: Proc. SPIE 4110 (2000) 9

4) Y. Ding, R. M. Brubaker, D. D. Nolte, M. R. Melloch and A. M. Weiner: Opt. Lett. 22 (1997) 718

5) M. C. Nuss, M. Li, T. H. Chiu, A. M. Weiner and A. Partovi: Opt. Lett. 19 (1994) 664.

6) K. Ema, J. Ishi, H. Kunugita, T. Ban and T. Kondo: Opt. \& Quantum Electron. 33 (2001) 1063

7) N. Peyghambarian, S. W. Koch and A. Mysyrowicz: Introduction to Semiconductor Optics (Prentice-Hall, 1993) p. 343. 